CALT-68-2091

hep-th/9701008

\title{
Coupling a Self-Dual Tensor to Gravity in Six Dimensions'
}

\author{
John H. Schwarz \\ California Institute of Technology, Pasadena, CA 91125, USA
}

\begin{abstract}
A recent result concerning interacting theories of self-dual tensor gauge fields in six dimensions is generalized to include coupling to gravity. The formalism makes five of the six general coordinate invariances manifest, whereas the sixth one requires a non-trivial analysis. The result should be helpful in formulating the world-volume action of the $\mathrm{M}$ theory five-brane.
\end{abstract}

\footnotetext{
${ }^{1}$ Work supported in part by the U.S. Dept. of Energy under Grant No. DE-FG03-92-ER40701.
} 
In a recent paper, Perry and the author studied interacting theories of a self-dual tensor gauge field in six dimensions [1]. Since there is no straightforward way to describe such a theory with manifest Lorentz invariance, we chose to use a formalism in which Lorentz invariance was manifest only in a $5 \mathrm{~d}$ subspace, while the remaining Lorentz symmetries were realized in a more subtle non-manifest way. The purpose of the present paper is to extend that work to include the coupling to a $6 \mathrm{~d}$ metric field. The resulting theories will have manifest general coordinate invariance in five directions and non-manifest general coordinate invariance in the sixth one.

There are two different motivations and potential applications of these results. The first is to $6 \mathrm{~d}$ supergravity theories. The minimal supergravity multiplet contains the self-dual tensor gauge field in addition to the graviton and gravitino fields. The second application is to the construction of the world-volume action of the $\mathrm{M}$ theory five-brane, since this is also a $6 \mathrm{~d}$ theory with a self-dual tensor gauge field. The metric in this case is induced from the superspace coordinates of the ambient 11d space-time.

In the following we denote $6 \mathrm{~d}$ coordinates by $x^{\hat{\mu}}=\left(x^{\mu}, x^{5}\right)$, where $\mu=0,1,2,3,4$. The $x^{5}$ direction is singled out as the one that will be treated differently from the other five. The $6 \mathrm{~d}$ metric $G_{\hat{\mu} \hat{\nu}}$ contains $5 \mathrm{~d}$ pieces $G_{\mu \nu}, G_{\mu 5}$, and $G_{55}$. All formulas will be written with manifest 5d general coordinate invariance. As in Ref. [1], we represent the self-dual tensor gauge field by a $5 \times 5$ antisymmetric tensor $B_{\mu \nu}$. The way this comes about can be described quite easily for the free theory. If one starts with a $6 \mathrm{~d}$ tensor $B_{\hat{\mu} \hat{\nu}}$, with field strength $H_{\hat{\mu} \hat{\nu} \hat{\rho}}=3 \partial_{[\hat{\mu}} B_{\hat{\nu} \hat{\rho}]}$, then the self-duality condition is the first-order field equation

$$
H_{\hat{\mu} \hat{\nu} \hat{\rho}}=\frac{1}{6 \sqrt{-G}} G_{\hat{\mu} \hat{\mu}^{\prime}} G_{\hat{\nu} \hat{\nu}^{\prime}} G_{\hat{\rho} \hat{\rho}^{\prime}} \epsilon^{\hat{\mu}^{\prime} \hat{\nu}^{\prime} \hat{\rho}^{\prime} \hat{\lambda} \hat{\sigma} \hat{\eta}} H_{\hat{\lambda} \hat{\sigma} \hat{\eta}},
$$

where $G=\operatorname{det} G_{\hat{\mu} \hat{\nu}}$. It is possible to solve this equation for $H_{\mu \nu 5}$ in terms of $G_{\hat{\mu} \hat{\nu}}$ and $H_{\mu \nu \rho}$ :

$$
H_{\mu \nu 5}=K_{\mu \nu}(G, H)
$$

Since

$$
H_{\mu \nu 5}=\partial_{5} B_{\mu \nu}+\partial_{\mu} B_{\nu 5}-\partial_{\nu} B_{\mu 5}
$$

taking a curl eliminates $B_{\mu 5}$ leaving a second-order field equation that involves $B_{\mu \nu}$ only:

$$
\frac{1}{2} \epsilon^{\mu \nu \rho \lambda \sigma} \partial_{\rho} K_{\lambda \sigma}=\frac{1}{2} \epsilon^{\mu \nu \rho \lambda \sigma} \partial_{5} \partial_{\rho} B_{\lambda \sigma}=\partial_{5} \tilde{H}^{\mu \nu}
$$

where

$$
\tilde{H}^{\mu \nu}=\frac{1}{6} \epsilon^{\mu \nu \rho \lambda \sigma} H_{\rho \lambda \sigma}
$$


It is a straightforward exercise to formulate a Lagrangian that gives the field equation (4). The result is $L=L_{1}+L_{2}+L_{3}$, where

$$
\begin{aligned}
L_{1} & =\frac{\sqrt{-G}}{2\left(-G_{5}\right)} \operatorname{tr}(G \tilde{H} G \tilde{H}), \\
L_{2} & =\frac{1}{2} \tilde{H}^{\mu \nu} \partial_{5} B_{\mu \nu}, \\
L_{3} & =-\frac{1}{4} \epsilon_{\mu \nu \rho \lambda \sigma} \frac{G^{5 \rho}}{G^{55}} \tilde{H}^{\mu \nu} \tilde{H}^{\lambda \sigma} .
\end{aligned}
$$

This result was given previously in Ref. [3]. The only difference is that there the time direction was treated as special, whereas now we are singling out a spatial direction. Note that $L_{2}$ contains the only $x^{5}$ derivative, and it does not depend on the metric. The notation is as follows: $G_{5}$ is the 5 d determinant $\left(G_{5}=\operatorname{det} G_{\mu \nu}\right)$, while $G^{55}$ and $G^{5 \rho}$ are components of the inverse $6 \mathrm{~d}$ metric $G^{\hat{\mu} \hat{\nu}}$. The trace only involves $5 \mathrm{~d}$ indices:

$$
\operatorname{tr}(G \tilde{H} G \tilde{H})=G_{\mu \nu} \tilde{H}^{\nu \rho} G_{\rho \lambda} \tilde{H}^{\lambda \mu}
$$

The $\epsilon$ symbols are purely numerical with $\epsilon^{01234}=1$ and $\epsilon^{\mu \nu \rho \lambda \sigma}=-\epsilon_{\mu \nu \rho \lambda \sigma}$. A useful relation is $G_{5}=G G^{55}$.

Our goal now is to generalize the preceding free theory result to the case of an interacting theory. For this purpose we consider the generalization

$$
L_{1}=\sqrt{-G} f\left(z_{1}, z_{2}\right)
$$

while keeping $L_{2}$ and $L_{3}$ unchanged. The $z$ variables are defined to be

$$
\begin{aligned}
z_{1} & =\frac{\operatorname{tr}(G \tilde{H} G \tilde{H})}{2\left(-G_{5}\right)} \\
z_{2} & =\frac{\operatorname{tr}(G \tilde{H} G \tilde{H} G \tilde{H} G \tilde{H})}{4\left(-G_{5}\right)^{2}} .
\end{aligned}
$$

This is quite general, since traces of higher powers of $G \tilde{H}$ can be reexpressed in terms of $z_{1}$ and $z_{2}$. The quantities $z_{1}$ and $z_{2}$ are scalars under 5 d general coordinate transformations. Note that the free theory result corresponds to $f=z_{1}$. Our goal now is to determine the class of functions $f$ for which the theory has $6 \mathrm{~d}$ general coordinate invariance.

Infinitesimal parameters of general coordinate transformations are denoted $\xi^{\hat{\mu}}=\left(\xi^{\mu}, \xi\right)$. Since $5 \mathrm{~d}$ general coordinate invariance is manifest, we focus on the $\xi$ transformations only. The metric transforms in the standard way

$$
\delta_{\xi} G_{\hat{\mu} \hat{\nu}}=\xi \partial_{5} G_{\hat{\mu} \hat{\nu}}+\partial_{\hat{\mu}} \xi G_{5 \hat{\nu}}+\partial_{\hat{\nu}} \xi G_{\hat{\mu} 5} .
$$


However, something non-standard is required for $B_{\mu \nu}$. If we had an ordinary $6 \mathrm{~d}$ tensor $B_{\hat{\mu} \hat{\nu}}$, the standard transformation would be $\delta B_{\hat{\mu} \hat{\nu}}=\xi^{\hat{\rho}} H_{\hat{\rho} \hat{\mu} \hat{\nu}}$, which gives, in particular, $\delta_{\xi} B_{\mu \nu}=$ $\xi H_{5 \mu \nu}$. But $H_{5 \mu \nu}$ contains fields that are not part of our theory. To see what is going on, let us form the $B_{\mu \nu}$ field equation. It is given again by eq. (四), where now

$$
K_{\mu \nu}=-\frac{\partial\left(L_{1}+L_{3}\right)}{\partial \tilde{H}^{\mu \nu}}=K_{\mu \nu}^{(1)} f_{1}+K_{\mu \nu}^{(2)} f_{2}+K_{\mu \nu}^{(\epsilon)}
$$

with

$$
\begin{aligned}
K_{\mu \nu}^{(1)} & =\frac{\sqrt{-G}}{\left(-G_{5}\right)}(G \tilde{H} G)_{\mu \nu} \\
K_{\mu \nu}^{(2)} & =\frac{\sqrt{-G}}{\left(-G_{5}\right)^{2}}(G \tilde{H} G \tilde{H} G \tilde{H} G)_{\mu \nu} \\
K_{\mu \nu}^{(\epsilon)} & =\epsilon_{\mu \nu \rho \lambda \sigma} \frac{G^{5 \rho}}{2 G^{55}} \tilde{H}^{\lambda \sigma},
\end{aligned}
$$

and we have defined

$$
f_{i}=\frac{\partial f}{\partial z_{i}}, \quad i=1,2 .
$$

There is now a natural guess for $\delta_{\xi} B_{\mu \nu}$, since the field equation implies that $H_{5 \mu \nu}$ is equivalent to $K_{\mu \nu}$ on shell. Therefore we postulate the transformation law

$$
\delta_{\xi} B_{\mu \nu}=\xi K_{\mu \nu} .
$$

The next step is to examine the symmetry by computing the variation of the action under the transformations $\delta_{\xi} G_{\hat{\mu} \hat{\nu}}$ and $\delta_{\xi} B_{\mu \nu}$ given in eqs. (10) and (14). We will speak of the variation of $L$, but since it is the action we are really interested in, total derivatives will be dropped whenever convenient. The variation is a sum of three pieces

$$
\delta L=\delta_{G}\left(L_{1}+L_{3}\right)+\delta_{B}\left(L_{1}+L_{3}\right)+\delta_{B} L_{2} .
$$

The $B$ variations are relatively simple:

$$
\begin{aligned}
\delta_{B}\left(L_{1}+L_{3}\right) & =\left(\delta_{\xi} \tilde{H}^{\mu \nu}\right) \frac{\partial\left(L_{1}+L_{3}\right)}{\partial \tilde{H}^{\mu \nu}}=-\frac{1}{2} \epsilon^{\mu \nu \rho \lambda \sigma} \partial_{\rho}\left(\xi K_{\lambda \sigma}\right) K_{\mu \nu} \sim-\frac{1}{4} \epsilon^{\mu \nu \rho \lambda \sigma} K_{\mu \nu} K_{\lambda \sigma} \partial_{\rho} \xi, \\
\delta_{B} L_{2} & \sim-\delta_{\xi} B_{\mu \nu} \partial_{5} \tilde{H}^{\mu \nu}=-\xi K_{\mu \nu} \partial_{5} \tilde{H}^{\mu \nu} .
\end{aligned}
$$

The symbol $\sim$ means that a total derivative has been dropped. Somewhat more complicated is

$$
\begin{aligned}
\delta_{G}\left(L_{1}+L_{3}\right)= & \partial_{5}(\xi \sqrt{-G}) f+\sqrt{-G} \delta_{G} f-\frac{1}{4} \epsilon_{\mu \nu \rho \lambda \sigma} \delta\left(\frac{G^{5 \rho}}{G^{55}}\right) \tilde{H}^{\mu \nu} \tilde{H}^{\lambda \sigma} \\
\sim & \sqrt{-G}\left(-\xi\left(f_{1} \partial_{5} z_{1}+f_{2} \partial_{5} z_{2}\right)+f_{1} \delta_{G} z_{1}+f_{2} \delta_{G} z_{2}\right) \\
& -\frac{1}{4} \epsilon_{\mu \nu \rho \lambda \sigma}\left[\partial_{5}\left(\xi \frac{G^{5 \rho}}{G^{55}}\right)+\partial_{\eta} \xi\left(\frac{2 G^{5 \eta} G^{5 \rho}}{\left(G^{55}\right)^{2}}-\frac{G^{\eta \rho}}{G^{55}}\right)\right] \tilde{H}^{\mu \nu} \tilde{H}^{\lambda \sigma} .
\end{aligned}
$$


Here one must insert

$$
\delta_{G} z_{1}=\xi \partial_{5} z_{1}+\xi\left(-G_{5}\right)^{-1} \partial_{5} \tilde{H}^{\mu \nu}(G \tilde{H} G)_{\mu \nu}+2 \partial_{\mu} \xi\left(\frac{G^{5 \mu}}{G^{55}} z_{1}+\frac{G_{5 \nu}}{\left(-G_{5}\right)}(\tilde{H} G \tilde{H})^{\mu \nu}\right)
$$

and

$$
\begin{aligned}
\delta_{G} z_{2}= & \xi \partial_{5} z_{2}+\xi\left(-G_{5}\right)^{-2} \partial_{5} \tilde{H}^{\mu \nu}(G \tilde{H} G \tilde{H} G \tilde{H} G)_{\mu \nu} \\
& +2 \partial_{\mu} \xi\left(\frac{2 G^{5 \mu}}{G^{55}} z_{2}+\frac{G_{5 \nu}}{\left(-G_{5}\right)^{2}}(\tilde{H} G \tilde{H} G \tilde{H} G \tilde{H})^{\mu \nu}\right) .
\end{aligned}
$$

Now we collect terms and simplify. For example, the terms proportional to $f_{1}$ are

$$
\begin{aligned}
& -\xi K_{\mu \nu}^{(1)} f_{1} \partial_{5} \tilde{H}^{\mu \nu}-\frac{1}{2} \epsilon^{\mu \nu \rho \lambda \sigma} \partial_{\rho} \xi K_{\mu \nu}^{(1)} f_{1} K_{\lambda \sigma}^{(\epsilon)}+\xi \frac{\sqrt{-G}}{\left(-G_{5}\right)} \partial_{5} \tilde{H}^{\mu \nu}(G \tilde{H} G)_{\mu \nu} f_{1} \\
& +2 \partial_{\mu} \xi \sqrt{-G}\left(\frac{G^{5 \mu}}{G^{55}} z_{1}+\frac{G_{5 \nu}}{\left(-G_{5}\right)}(\tilde{H} G \tilde{H})^{\mu \nu}\right) f_{1} .
\end{aligned}
$$

The first and third terms, which are proportional to $\xi$, cancel. To compare the other two terms we use

$$
\epsilon^{\mu \nu \rho \lambda \sigma} K_{\lambda \sigma}^{(\epsilon)}=-\delta_{\mu^{\prime} \nu^{\prime} \rho^{\prime}}^{\mu \nu \rho} \frac{G^{5 \rho^{\prime}}}{G^{55}} \tilde{H}^{\mu^{\prime} \nu^{\prime}}
$$

Then, using $G^{5 \mu} G_{\mu \nu}=-G^{55} G_{5 \nu}$, it follows that the second and fourth terms also cancel. The terms proportional to $f_{2}$ cancel in the same way.

The remaining terms proportional to $\xi$ are $-\xi K_{\mu \nu}^{(\epsilon)} \partial_{5} \tilde{H}^{\mu \nu}$ and the term that results from partially integrating the $\partial_{5}$ term in the last line of eq. (17). These two terms cancel.

The terms that now remain are all proportional to $\partial_{\mu} \xi$ :

$$
\begin{gathered}
\frac{1}{4} \epsilon_{\mu \nu \rho \lambda \sigma} \partial_{\eta} \xi\left(\frac{G^{\eta \rho}}{G^{55}}-2 \frac{G^{5 \eta} G^{5 \rho}}{\left(G^{55}\right)^{2}}\right) \tilde{H}^{\mu \nu} \tilde{H}^{\lambda \sigma}-\frac{1}{4} \epsilon^{\mu \nu \rho \lambda \sigma} \partial_{\rho} \xi K_{\mu \nu}^{(\epsilon)} K_{\lambda \sigma}^{(\epsilon)} \\
-\frac{1}{4} \epsilon^{\mu \nu \rho \lambda \sigma} \partial_{\rho} \xi\left(K_{\mu \nu}^{(1)} f_{1}+K_{\mu \nu}^{(2)} f_{2}\right)\left(K_{\lambda \sigma}^{(1)} f_{1}+K_{\lambda \sigma}^{(2)} f_{2}\right) .
\end{gathered}
$$

The two terms on the first line of this expression combine to give

$$
W=\epsilon_{\mu \nu \rho \lambda \sigma} \partial_{\eta} \xi \frac{G_{5}^{\eta \rho}}{4 G^{55}} \tilde{H}^{\mu \nu} \tilde{H}^{\lambda \sigma}
$$

where

$$
G_{5}^{\eta \rho}=G^{\eta \rho}-\frac{G^{5 \eta} G^{5 \rho}}{G^{55}}
$$

is the inverse of the $5 \times 5$ matrix $G_{\eta \rho}$. 
It remains to evaluate the terms on the second line of (22). Let us begin with the term proportional to $f_{1}^{2}$. Using the identity

$$
\epsilon^{\mu \nu \rho \lambda \sigma} G_{\mu \mu^{\prime}} G_{\nu \nu^{\prime}} G_{\lambda \lambda^{\prime}} G_{\sigma \sigma^{\prime}}=-G_{5} \epsilon_{\mu^{\prime} \nu^{\prime} \rho^{\prime} \lambda^{\prime} \sigma^{\prime}} G_{5}^{\rho \rho^{\prime}}
$$

one finds that

$$
-\frac{1}{4} \epsilon^{\mu \nu \rho \lambda \sigma} \partial_{\rho} \xi K_{\mu \nu}^{(1)} K_{\lambda \sigma}^{(1)} f_{1}^{2}=-f_{1}^{2} W .
$$

Using the same identity in the $f_{1} f_{2}$ term gives

$$
-\frac{1}{2} \epsilon^{\mu \nu \rho \lambda \sigma} \partial_{\rho} \xi K_{\mu \nu}^{(1)} K_{\lambda \sigma}^{(2)} f_{1} f_{2}=-\frac{1}{2} f_{1} f_{2} \frac{(-G)}{\left(-G_{5}\right)^{2}} \epsilon_{\rho^{\prime} \mu \nu \lambda \sigma} \partial_{\rho} \xi G_{5}^{\rho \rho^{\prime}} \tilde{H}^{\mu \nu}(\tilde{H} G \tilde{H} G \tilde{H})^{\lambda \sigma} .
$$

To simplify this further, we consider the factor

$$
\epsilon_{\rho^{\prime} \mu \nu \lambda \sigma} G_{5}^{\rho \rho^{\prime}} \tilde{H}^{\mu \nu} \tilde{H}^{\lambda \eta} \tilde{H}^{\zeta \sigma}
$$

which we represent by $\left(\rho^{\prime} \mu \nu \lambda \eta \sigma\right)$ to indicate the sequence in which these six superscripts appear. Because antisymmetrization of six 5-valued indices gives zero, one has the relation

$$
\left(\rho^{\prime} \mu \nu \lambda \eta \sigma\right)=\left(\sigma \rho^{\prime} \mu \nu \lambda \eta\right)-\left(\eta \sigma \rho^{\prime} \mu \nu \lambda\right)+\left(\lambda \eta \sigma \rho^{\prime} \mu \nu\right)-\left(\nu \lambda \eta \sigma \rho^{\prime} \mu\right)+\left(\mu \nu \lambda \eta \sigma \rho^{\prime}\right) .
$$

The second term on the right-hand side of eq. (29) contains

$$
\epsilon_{\rho^{\prime} \mu \nu \lambda \sigma} \tilde{H}^{\sigma \rho^{\prime}} \tilde{H}^{\mu \nu} \tilde{H}^{\zeta \lambda}
$$

The antisymmetry of $\tilde{H}$ and the fact that the indices are 5-valued implies that this vanishes. The last three terms on the right-hand side of (29) are each equal to the negative of the expression on the left. Thus

$$
\left(\rho^{\prime} \mu \nu \lambda \eta \sigma\right)=\frac{1}{4}\left(\sigma \rho^{\prime} \mu \nu \lambda \eta\right)
$$

Substituting this into eq. (27) gives

$$
-\frac{1}{8} f_{1} f_{2} \frac{(-G)}{\left(-G_{5}\right)^{2}} \epsilon_{\rho^{\prime} \mu \nu \lambda \sigma} \partial_{\rho} \xi G_{5}^{\rho \sigma} \tilde{H}^{\rho^{\prime} \mu} \tilde{H}^{\nu \lambda}(G \tilde{H} G)_{\eta \zeta} \tilde{H}^{\zeta \eta}=-z_{1} f_{1} f_{2} W .
$$

The term proportional to $f_{2}^{2}$ is evaluated by the same methods and gives

$$
\left(\frac{1}{2} z_{1}^{2}-z_{2}\right) f_{2}^{2} W
$$

Assembling the results given above, the required general coordinate transformation symmetry is achieved if, and only if, the function $f$ satisfies the nonlinear partial differential equation

$$
f_{1}^{2}+z_{1} f_{1} f_{2}+\left(z_{2}-\frac{1}{2} z_{1}^{2}\right) f_{2}^{2}=1
$$


This is precisely the same equation that was derived in Ref. [3] as the condition for self duality of a 4d U(1) gauge theory and in Ref. [1] as the condition for Lorentz invariance of the $6 \mathrm{~d}$ theory with $G_{\hat{\mu} \hat{\nu}}=\eta_{\hat{\mu} \hat{\nu}}$. As discussed in [1], it has many solutions, but the two solutions of most interest are the "free" $\left(f=z_{1}\right)$ solution and the "Born-Infeld" solution

$$
f=2 \sqrt{1+z_{1}+\frac{1}{2} z_{1}^{2}-z_{2}} .
$$

The former gives the bosonic part of the $6 \mathrm{~d}$ supergravity action and the latter gives the bosonic part of the $\mathrm{M}$ theory five-brane action. In the latter case one can reexpress the complete bosonic Lagrangian as

$$
L=2 \sqrt{-\operatorname{det}\left(G_{\hat{\mu} \hat{\nu}}+i G_{\hat{\mu} \rho} G_{\hat{\nu} \lambda} \tilde{H}^{\rho \lambda} / \sqrt{-G_{5}}\right)}+\frac{1}{2} \tilde{H}^{\mu \nu} \partial_{5} B_{\mu \nu}-\frac{1}{4} \epsilon_{\mu \nu \rho \lambda \sigma} \frac{G^{5 \rho}}{G^{55}} \tilde{H}^{\mu \nu} \tilde{H}^{\lambda \sigma} .
$$

All that remains to construct the $\mathrm{M}$ theory five-brane action is to add the appropriate dependences on fermionic degrees of freedom and to establish local kappa symmetry. The result will be reported elsewhere [4].

I am grateful to M. Aganagic and C. Popescu for helpful discussions.

\section{References}

[1] M. Perry and J.H. Schwarz, "Interacting Chiral Gauge Fields in Six Dimensions and Born-Infeld Theory," hep-th/9611065.

[2] J.H. Schwarz and A. Sen, Nucl. Phys. B411 (1994) 35, hep-th/9304154.

[3] G.W. Gibbons and D.A. Rasheed, Nucl. Phys. B454 (1995) 185, hep-th/9506035.

[4] M. Aganagic, J. Park, C. Popescu, and J.H. Schwarz, to appear. 